\title{
NOUVELLE
}

\section{Une nouvelle forme d'amaurose congénitale de Leber}

Défaillance de la neuroprotection

des photorécepteurs et des cellules ganglionnaires de la rétine

Josseline Kaplan, Isabelle Perrault, Sylvain Hanein, Hélène Dollfus, Jean-Michel Rozet
Unité de recherche génétique et épigénétique des maladies métaboliques, neurosensorielles et du développement, Inserm U781, hôpital Necker-Enfants malades, tour Lavoisier, 149, rue de Sèvres, 75015 Paris Cedex 15, France. josseline.kaplan@inserm.fr

\section{L'amaurose congénitale de Leber} L'amaurose congénitale de Leber ( $A C L$ ) désigne un groupe de dystrophies rétiniennes précoces et sévères, survenant dans les premiers mois de vie et entraînant une malvoyance profonde [1-3]. Elle représente 10 à $18 \%$ des cécités de l'enfance. Au cours des vingt dernières années, cette affection de transmission récessive autosomique a révélé une variabilité clinique inattendue : on distingue un type 1 , dramatiquement sévère et stationnaire par atteinte cônes-bâtonnets (cone-rod dystrophy), et un type 2, certes sévère mais évolutif, par atteinte bâtonnets-cônes (rod-cone dystrophy), considéré comme l'extrémité du spectre de gravité des rétinopathies pigmentaires [2]. Au demeurant, alors que I'ACL avait été considérée pendant plus d'un siècle comme une malvoyance isolée, de nombreuses observations de manifestations extraoculaires associées ont suggéré l'existence de formes syndromiques, que le décryptage génétique a permis d'intégrer dans la famille sans cesse croissante des ciliopathies. En outre, l'hétérogénéité génétique, suspectée depuis les années 1950, a dépassé toutes les prévisions par la mise en évidence de l'implication de 18 gènes couvrant, tous ensemble, $70 \%$ des cas d'ACL. Enfin, une hétérogénéité physiopathologique surprenante a été mise en lumière avec l'implication de mécanismes très divers, en particulier des anomalies de la transduction visuelle, du développement des photorécepteurs, du métabolisme de la vitamine $A$ et du transport intraflagellaire.

\section{Mutations du gène NMNATI}

Afin de poursuivre l'identification des bases moléculaires de cette affection pour laquelle nous avons un recensement exceptionnel de patients, nous avons soumis l'ADN de cinq patients dont l'étude génétique avait exclu l'implication de tous les gènes d'ACL connus à un séquençage de l'exome (projet « 100 exomes » financé par l'association RETINA, France). Quatre variants nucléotidiques du gène NMNATl (nuclear nicotinamide mononucleotide adenyltransferase 1) ont été identifiés chez deux patients [4]. Le séquençage (méthode de Sanger) de ces variants a confirmé la ségrégation familiale dans les deux cas et exclu la présence de ces variants chez 200 contrôles.

Nous avons ensuite criblé le gène NMNATI (exons et sites d'épissage) dans une cohorte de 256 patients et identifié chez 20 patients supplémentaires des mutations de ce gène, à l'état homozygote ou hétérozygote composite, non retrouvées dans la population contrôle ; chez sept autres patients, une seule mutation hétérozygote a été mise en évidence. L'une de ces mutations, p.£257K, a été retrouvée très fréquemment sur un des allèles (17 des 22 patients ont deux allèles mutés, un patient est hétérozygote). Chez 12 de ces 18 patients non apparentés $(66,7 \%)$, la mutation était liée à l'allèle 279 pb du marqueur D1S244, distant de 0,5 Mb, allèle quasi inexistant dans la population générale, suggérant que cette mutation a pu survenir sur un allèle ancestral.

NMNATI code l'isoforme nucléaire de la mononucléotide nicotinamide adényltransférase, acteur clé de la synthèse du NAD. Cette enzyme homohexamérique catalyse la formation de NAD à partir de nicotinamide mononucléotide (NMN) et d'ATP. L'effet des mutations sur la structure tridimensionnelle de l'enzyme a été analysé à l'aide du programme Swiss-Pdb Wiewer 3.7. Les résultats de cette modélisation suggèrent un effet délétère des mutations sur l'activité de l'enzyme.

Les 29 patients porteurs de mutations du gène NMNATI (y compris les sept hétérozygotes simples) sont atteints d'une amaurose congénitale de Leber de type 1, très sévère. Le phénotype oculaire est très particulier : il existe très précocement une atrophie de la région centrale de la rétine, rapidement évolutive, pouvant être considérée comme un colobome maculaire. Cette dégénérescence rétinienne centrale s'associe à une atrophie du nerf optique elle aussi inhabituellement très précoce, et ces deux signes permettent une reconnaissance extrêmement rapide de cette nouvelle forme d'ACL (Figure 1).

\section{Autres isoformes de NMNAT chez \\ l'homme et l'animal}

Deux autres isoformes de NMNAT existent : NMNAT2, localisée dans l'appareil de 


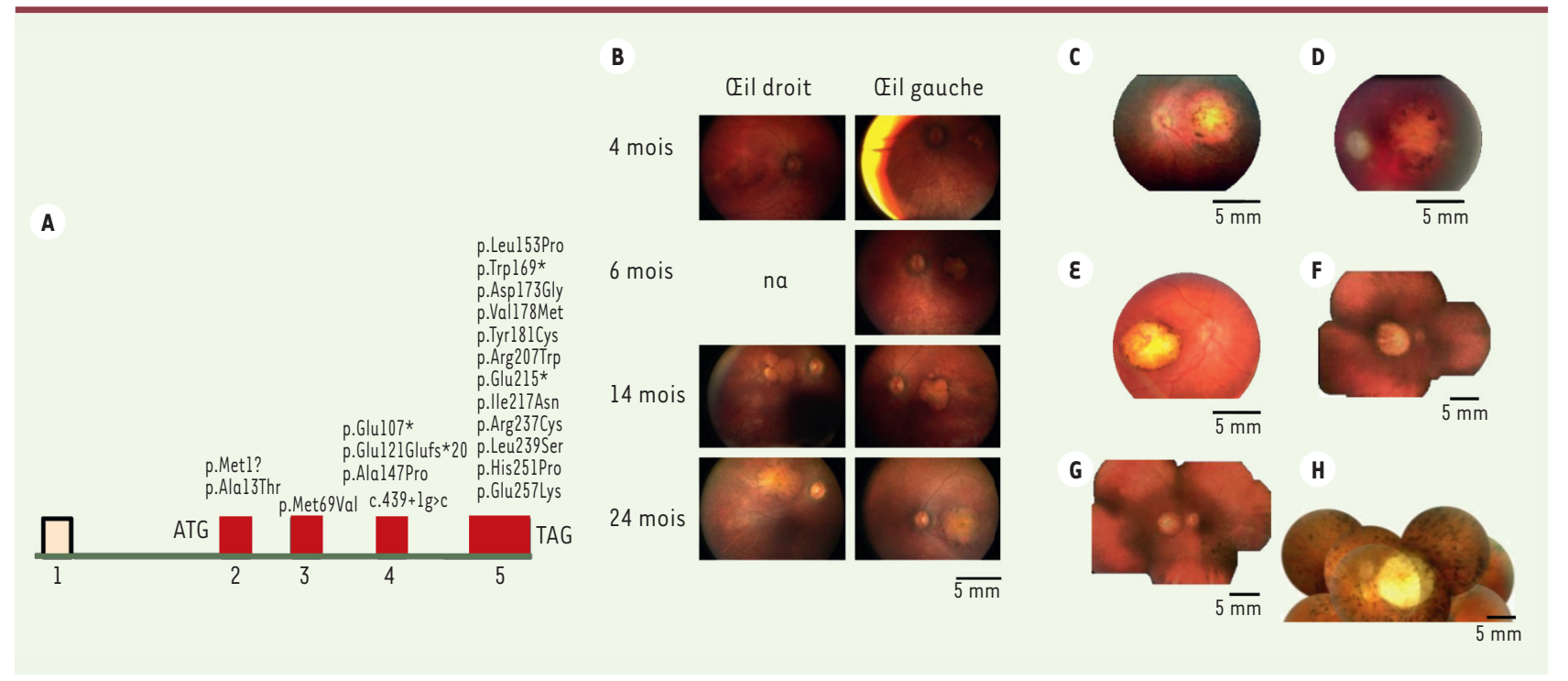

Figure 1. Amaurose congénitale de Leber due aux mutations du gène NMNAT1. A. Structure du gène NMNAT1 et position des mutations identifiées. B. Aspect ophtalmoscopique des lésions chez les patients. Progression des lésions maculaires d'un patient entre 4 et 24 mois après la naissance montrant la survenue et la progression rapide de l'atrophie maculaire. C-H. Aspects du fond d'œil chez six autres patients âgés de 4 à 20 ans. À noter l'aspect pseudo-colobomateux et l'atrophie optique (extrait de [4], (c) Nature Publishing Group, 2012).

Golgi, et NMNAT3, dans la mitochondrie. Chez la souris, l'invalidation de NMNATI est létale, indiquant que les deux autres isoformes ne peuvent se substituer à NMNATI. Chez la drosophile, l'inactivation de l'unique isoforme, nmnat, est également létale. Lorsque l'inactivation est restreinte aux photorécepteurs, elle entraîne une dégénérescence rétinienne très précoce survenant dans des photorécepteurs normalement développés. La perte des photorécepteurs apparaît déclenchée par l'exposition à la lumière, ce qui suggère que la dégénérescence est aggravée par l'activité neuronale. Chez le patient humain, les atteintes rétiniennes sont très superposables à celles de la drosophile, puisque l'atrophie maculaire et l'atrophie du nerf optique, absentes à la naissance, se développent très rapidement après la mise en route du processus de phototransduction ${ }^{1}$.

NMNATI est un élément majeur du complexe protéique Wallerian degeneration slow (WIds) qui retarde la dégénérescence axonale après une blessure des fibres nerveuses périphériques ou

\footnotetext{
${ }^{1}$ Les mécanismes de phototransduction permettent la
} conversion de l'énergie lumineuse en message nerveux. centrales. Plusieurs études ont montré que l'activité catalytique de NMNATI était nécessaire, mais non suffisante, pour une totale protection neuronale. Chez la drosophile, la protéine privée de son action enzymatique protège de la dégénérescence des photorécepteurs matures, suggérant une action chaperonne de l'enzyme, indépendante de l'activité enzymatique obligatoire pour la survie de l'animal.

Les données génétiques et les résultats d'analyse structurale sont compatibles avec une activité résiduelle de l'enzyme NMNAT chez les patients atteints d'ACL, entraînant une mort rapide des photorécepteurs après exposition à la lumière, anomalies superposables à celles qui sont observées chez la drosophile [4].

\section{Conclusion}

Nous avons donc identifié un nouveau gène, dont les mutations sont responsables de la forme la plus précoce et sévère des dystrophies rétiniennes, qui s'ajoute à la liste déjà longue des acteurs de cette affection. Mais surtout, nous rapportons un nouveau mécanisme physiopathologique, la défaillance du système de protection contre la dégé- nérescence neuronale qui se traduit par une atrophie postnatale mais très rapide de la région maculaire de la rétine donnant un aspect pseudo-colobomateux, et surtout une atrophie optique dont la précocité est très inhabituelle dans cette affection. $\diamond$

\section{Mutations in NMNATl cause Leber}

congenital amaurosis with severe macular and optic atrophy

\section{LIENS D'INTÉRÊT}

Les auteurs déclarent n'avoir aucun lien d'intérêt concernant les données publiées dans cet article.

\section{RÉFÉRENCES}

1. Hanein S, Perrault I, Gerber S, et al. Leber congenital amaurosis: comprehensive survey of the genetic heterogeneity, refinement of the clinical definition, and genotype-phenotype correlations as a strategy for molecular diagnosis. Hum Mutat 2004; 23 : 306-17.

2. Kaplan J. Leber congenital amaurosisi from darkness to spotlight. Ophthalmic Genet 2008 ; 29 : 92-8.

3. Perrault I, Hanein S, Kaplan J. L'amaurose congénitale de Leber : les rétinol-déshydrogénases au banc des accusés. Med Sci (Paris) $2004 ; 20$ : 1066-8.

4. Perrault I, Hanein S, Zanlonghi X, et al. Mutations in NMNATl cause Leber congenital amaurosis with earlyonset severe macular and optic atrophy. Nat Genet $2012 ; 44: 975-7$.

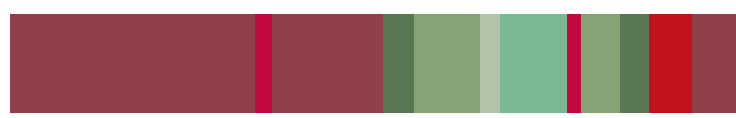

